\title{
Thoracoscopic resection of an ectopic mediastinal parathyroid adenoma in an octogenarian with recurrent hyperparathyroidism
}

\author{
Sengyi Deng ${ }^{1,2}$, Chengwu Liu ${ }^{1,2}$, Qiang Pu ${ }^{1,2}$, Lunxu Liu ${ }^{1,2}$ \\ ${ }^{1}$ Department of Thoracic Surgery, West China Hospital, Sichuan University, Chengdu 610041, China; ${ }^{2}$ Western China Collaborative Innovation \\ Center for Early Diagnosis and Multidisciplinary Therapy of Lung Cancer, Chengdu 610041, China \\ Correspondence to: Lunxu Liu, MD, PhD. No. 37, Guoxue Alley, Chengdu 610041, China. Email: lunxu_liu@aliyun.com.
}

\begin{abstract}
Primary hyperparathyroidism (HPT) is usually caused by hyper functioning parathyroid adenoma, whereas recurrent HPT caused by ectopic posterior mediastinal lesions is extremely rare. Herein, we present a case of an octogenarian with recurrent HPT after previous parathyroidectomy caused by a posterior mediastinal parathyroid adenoma. The ectopic parathyroid adenoma was successfully resected via thoracoscopic surgery and the patient completely recovered from HPT after operation. Our case demonstrated that surgical resection was effective for the treatment of recurrent HPT caused by ectopic mediastinal lesions and thoracoscopic approach was an optimal way for patients with compromised physical status.
\end{abstract}

Keywords: Hyperparathyroidism (HPT); parathyroid hormone (PTH); ectopic parathyroid adenoma; thoracoscopic surgery

Submitted Aug 08, 2018. Accepted for publication Aug 20, 2018.

doi: $10.21037 /$ tcr.2018.08.27

View this article at: http://dx.doi.org/10.21037/tcr.2018.08.27

\section{Introduction}

Primary hyperparathyroidism (HPT) is characterized by excessive secretion of parathyroid hormone (PTH), which is caused by hyper functioning parathyroid tissues, and is commonly accompanied with hypercalcaemia and various symptoms (1-4). Parathyroid adenomas are the leading causes of primary HPT (85\%), while other causes (15\%) are related to parathyroid hyperplasia and carcinoma (5). The optimal treatment for HPT is surgical resection of the pathological tissue (1). The surgical approaches varied according to the different location of the lesions (6). Although most parathyroid adenomas are located in the neck region, $11 \%$ to $25 \%$ of them are ectopically located within the mediastinum, among which ectopic location at the posterior mediastinum was extremely rare $(7,8)$. For mediastinal ectopic parathyroid adenomas, minimally invasive thoracoscopic approaches are now preferred (6,7,9-11). Here, we present our experience of surgical treatment in an octogenarian who suffered recurrent HPT, hypercalcaemia, and extremely severe osteoporosis caused by an ectopic parathyroid adenoma located posterior to the esophagus.

\section{Case presentation}

An 86-year-old woman had a history of HPT caused by parathyroid adenoma 5 years before admission. Surgical resection had been performed to remove the tumor. After the operation she was administered with Fosamax for about 3 months. Periodic reviews showed normal level of PTH and blood calcium. Eleven months before admission she complained of mild to moderate arthralgia. Three months before admission, biochemistry results showed high level of blood calcium and PTH: $3.05 \mathrm{mmol} / \mathrm{L}, \mathrm{PTH}$ : $26.78 \mathrm{pmol} / \mathrm{L}$, low level of serum phosphorus: $0.69 \mathrm{mmol} / \mathrm{L}$ and normal level of alkaline phosphatase (ALP): 77 IU/L (reference range: serum calcium, 2.1-2.7 mmol/L; PTH, 1.60-6.90 pmol/L; serum phosphorus, 0.81-1.45 mmol/L; ALP, 50-135 IU/L). Bone mineral density examinations revealed severe osteoporosis. Technetium (99mTc) sestamibi scintigraphy showed focal intense uptake and retention of 

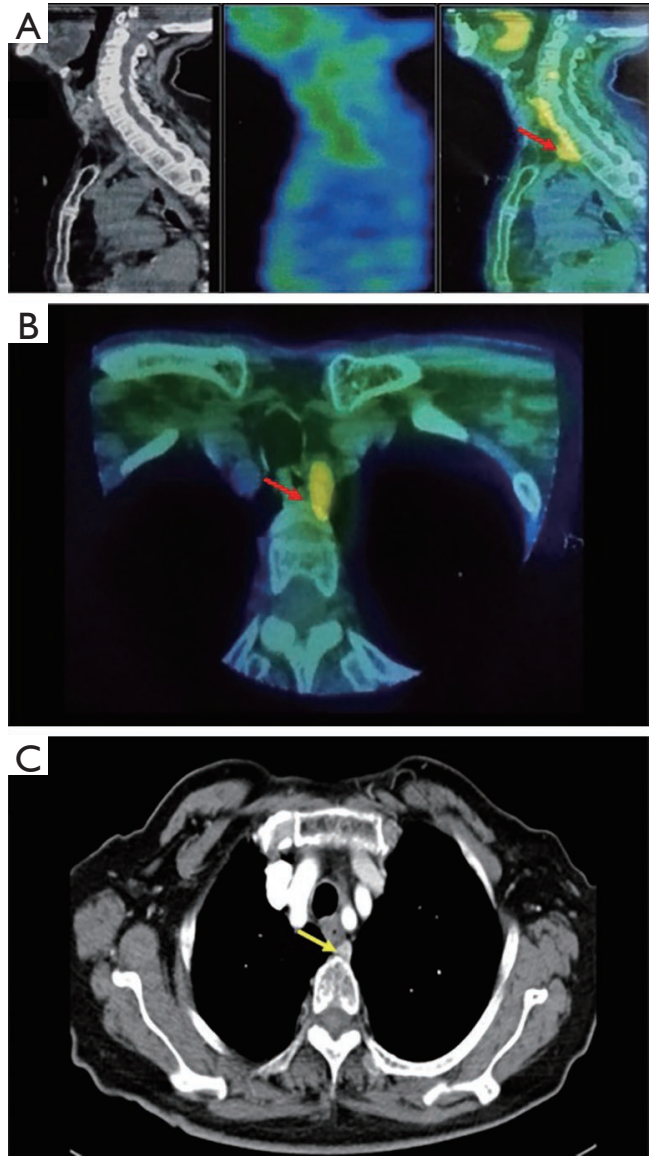

Figure 1 Technetium-99m sestamibi scintigraphy showing an intense uptake and retention of tracer in the left side of the mediastinum [red arrow, (A) coronal section, (B) sagittal section]. (C) Enhanced CT image of the thorax showing a well-defined mass in the posterior mediastinum just posterior to the esophagus (yellow arrow).

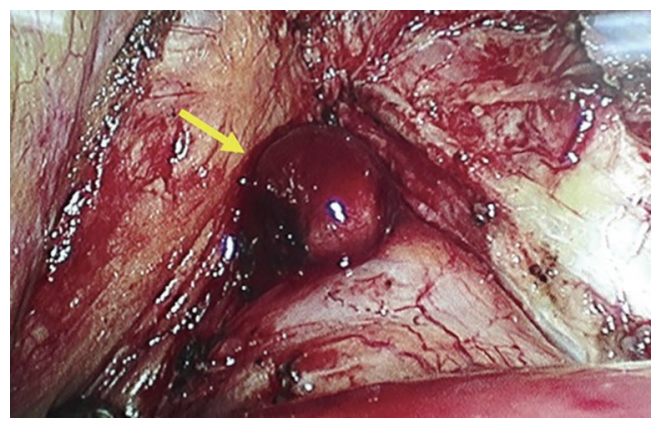

Figure 2 Intraoperative exploration demonstrating a well-defined mass posterior to the esophagus (yellow arrow). tracer in the posterior mediastinum (Figure 1A,B). Contrast enhanced computed tomography (CT) was performed for anatomic localization of the mass, which was determined to be in close contact with the esophagus (Figure 1C). Diagnosis was recurrent HPT caused by a hyper functioning ectopic parathyroid mass in the mediastinum. Besides, she had several comorbidities: primary hypertension, type II diabetes, chronic obstructive pulmonary disease, and coronary arteriosclerotic cardiopathy. Due to the poor physical condition, medical therapy was chosen to alleviate her symptoms. However, conservative therapy did not work well, her arthralgia became more and more severe. Before admission she could not walk and sit in a wheelchair. Thus, surgical resection became the last resort.

Video-assisted thoracoscopic surgery was chosen to minimize surgical trauma. Placed the patient in a lateral thoracotomy position, we performed a three-port thoracoscopic surgery under general anesthesia using double-lumen tracheal intubation. The observation port located in the seventh intercostal space at the midaxillary line, and two utility ports were placed in the third intercostal space at the anterior axillary line and the eighth intercostal space between posterior axillary line and subscapular line, respectively. The surgeon stood on the ventral side. The dissection was initiated by incision of the mediastinal pleura in the triangle among the left arteriae subclavian, aortic arch, and the spine. Intraoperative exploration revealed a well-defined mass $(2 \mathrm{~cm} \times 1.5 \mathrm{~cm})$ posterior to the esophagus (Figure 2), consistent with preoperative imaging studies. The tumor was completely resected using electrocautery and harmonic. The operation took only about 30 minutes with blood loss less than $10 \mathrm{~mL}$.

On postoperative day 1, calcium and PTH in serum were monitored with levels falling to $2.06 \mathrm{mmol} / \mathrm{L}$ and $8.85 \mathrm{pmol} / \mathrm{L}$, respectively. The obvious drop of the calcium and PTH confirmed success of the resection. The patient was discharged 8 days after surgery without any complications. Pathological examination revealed a parathyroid adenoma (Figure 3). She continued to have follow-up examinations in our out-patient clinic. Her arthralgia was palliated obviously and she could walk again three months after surgery, and blood test confirmed normal serum levels of calcium, PTH and phosphorus (Figure 4).

\section{Discussion}

Primary HPT often causes metabolic bone disease characterized by hypercalcaemia due to autonomous 

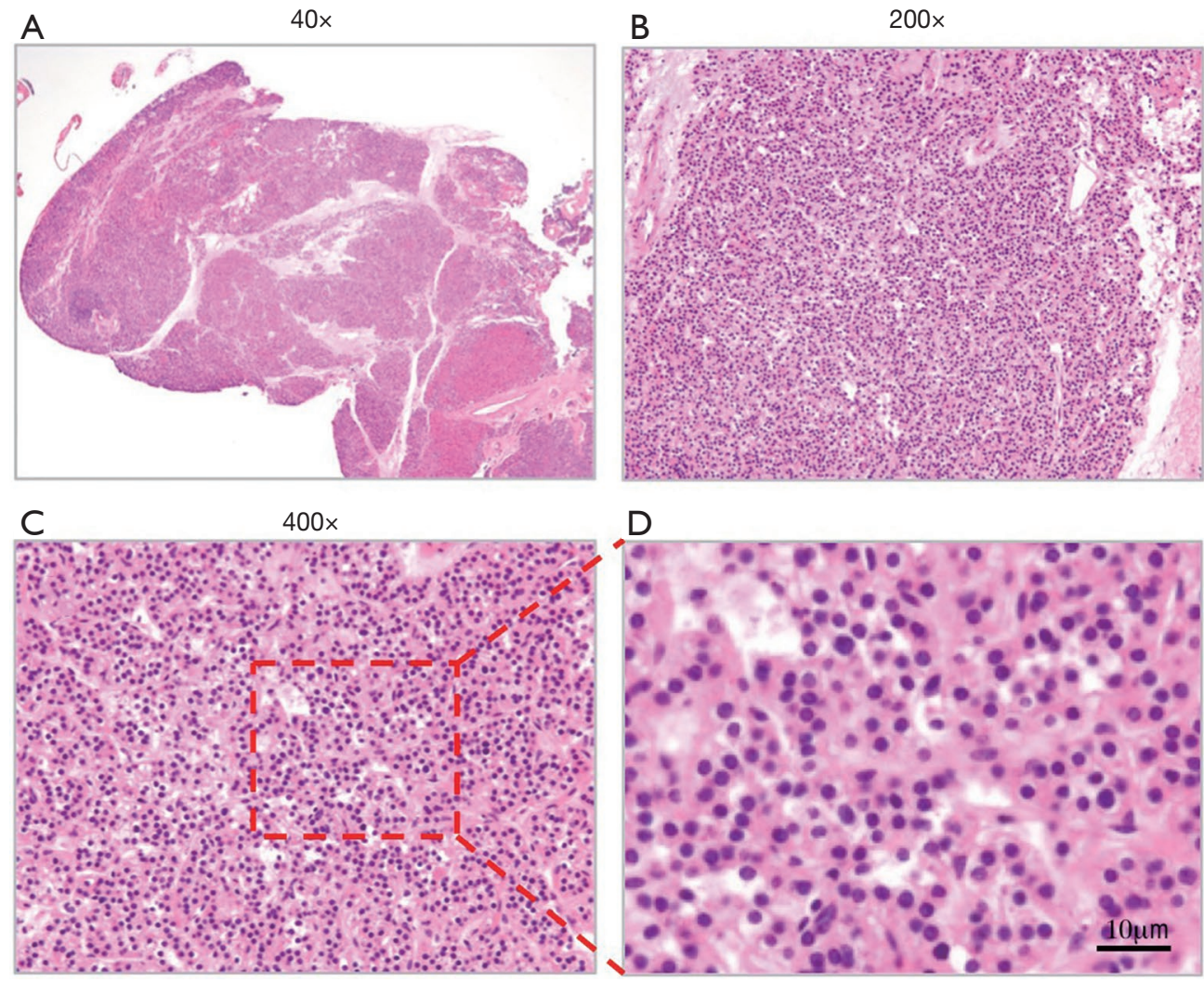

Figure 3 Haematoxylin and eosin staining of the pathological sections. (A) 40x; (B) 200x; (C) 400×; (D) magnified.

overproduction of PTH $(2,4)$. Most patients are asymptomatic while the others often suffered from ostealgia, arthralgia, tachycardia, urolithiasis, and so on, due to the presence of hypercalcaemia as demonstrated in our case $(1,3,4)$.

Though uncommon, ectopic location of active parathyroid pathologic tissue is an important reason in cases of disease recurrence or failed surgery $(1,7,12)$. In our case, we'd be pretty sure that the ectopic parathyroid adenoma contributing to the patient's disease recurrence. For symptomatic cases, surgical resection was suggested when medical treatment became ineffective. Previous studies reported that up to $25 \%$ of primary HPT was caused by ectopic mediastinal parathyroid glands, among which only about $2 \%$ are not accessible through lower anterior cervical approach $(6-8,13)$. Our case was a rare one with the lesion located in posterior mediastinum, which could not be approached through cervical approach. Since the first case of thoracoscopic excision of a mediastinal parathyroid by Prinz et al. in 1994 (14), many case series have documented safety and feasibility in using the same approach with overall low morbidity and mortality (7,9-11). Nevertheless, the success of thoracoscopy is dependent on accurate preoperative localization. Since there is not available an imaging investigation with $100 \%$ sensibility and specificity for identifying an ectopic parathyroid adenoma (15), we suggest for the imaging diagnostic procedure to include a combination of one functional and one structural investigation. Together with the experience reported in literature, we recommend CT plus Technetium (99mTc) sestamibi scintigraphy as a standard imaging investigation for a suspected ectopic mediastinal parathyroid adenoma (16).

It has been reported that intraoperative intact serum PTH assays was helpful for confirming a successful surgery $(17,18)$. As reported a reduction of PTH by at least $50 \%$ within ten minutes of excision strongly suggested a successful excision of an ectopic parathyroid adenomas $(18,19)$. We did not perform an intraoperative PTH assay, because the lesion was definite and identical to the imaging studies. What's more, considering the patient's age and poor physical status with such a lot of comorbidities, we'd like to complete the operation as fast as we could to reduce risks 

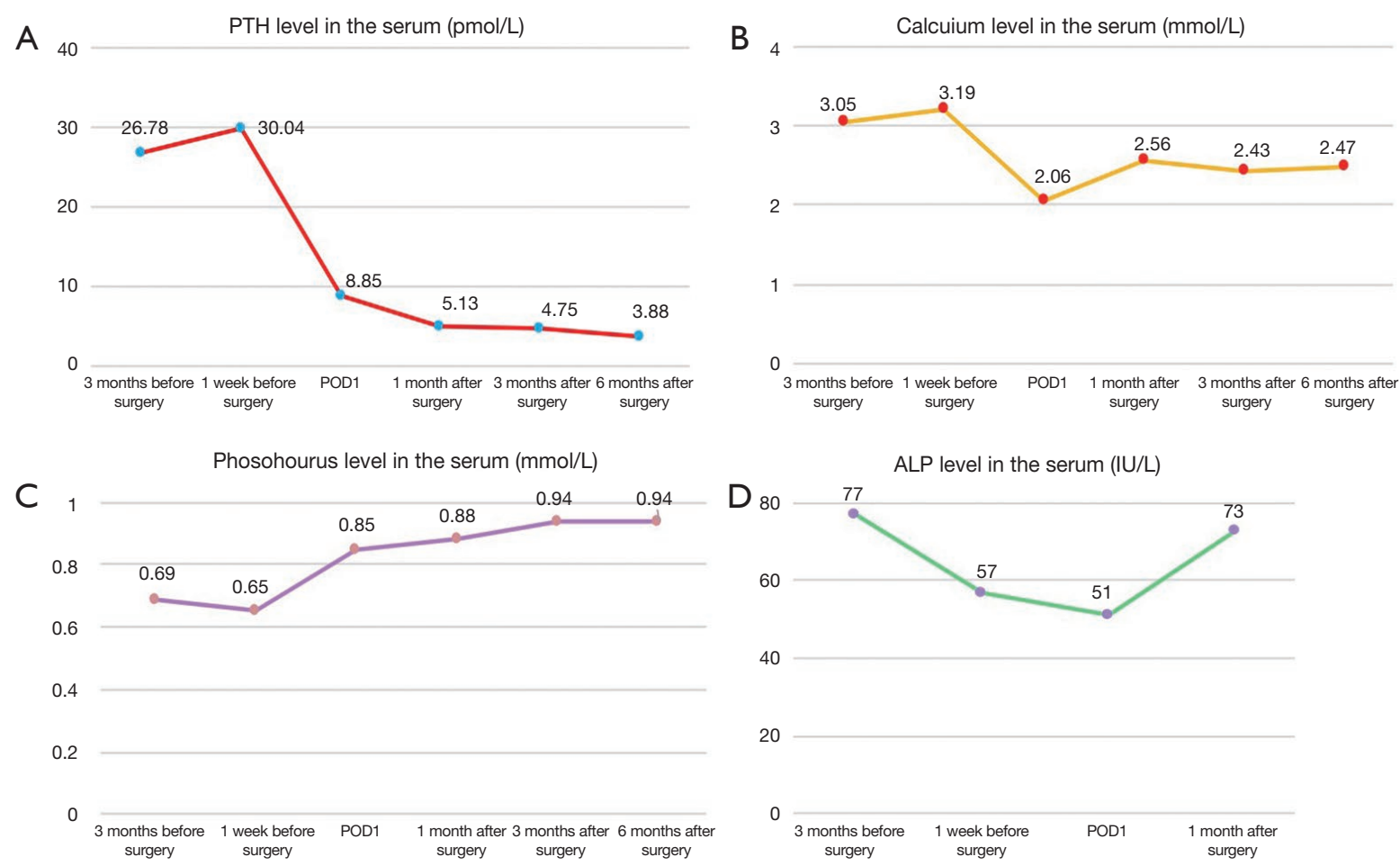

Figure 4 Perioperative serum levels of calcium (A), PTH (B), serum phosphorus (C) and alkaline phosphatase (D), reference range: serum calcium, 2.1-2.7 mmol/L; PTH, 1.60-6.90 pmol/L; serum phosphorus, 0.81-1.45 mmol/L; alkaline phosphatase, 50-135 IU/L.

of the operation. A sample taken on postoperative day 1 showed a remarkable reduction of PTH level, implying a complete removal of the hyperactive adenoma.

\section{Conclusions}

In this case report, we described an ectopic parathyroid adenoma in the posterior mediastinum of an octogenarian after parathyroidectomy, which was extremely rare. This case was unique and should be laid into the wide spectrum of recurrent HPT caused by ectopic parathyroid adenoma due to its rare location, compromised physical status, minimally invasive procedure, and good result of surgical resection. Our successful attempt demonstrated that surgical resection was effective for the treatment of recurrent HPT caused by ectopic mediastinal lesions and thoracoscopic approach was an optimal way for patients with compromised physical status.

\section{Acknowledgments}

Funding: This study was supported by National Natural
Science Foundation of China (No. 81372505 to L Liu) and (No. 81402240 to S Deng).

\section{Footnote}

Conflicts of Interest: TAll authors have completed the ICMJE uniform disclosure form (available at http://dx.doi. org/10.21037/tcr.2018.08.27). The authors have no conflicts of interest to declare.

Ethical statement: The authors are accountable for all aspects of the work in ensuring that questions related to the accuracy or integrity of any part of the work are appropriately investigated and resolved. All procedures performed in study involving human participants were in accordance with the ethical standards of the institutional and/or national research committee(s) and with the Declaration of Helsinki (as revised in 2013). Written informed consent was obtained from the patient's guardian for publication of this manuscript and any accompanying images.

Open Access Statement: This is an Open Access article 
distributed in accordance with the Creative Commons Attribution-NonCommercial-NoDerivs 4.0 International License (CC BY-NC-ND 4.0), which permits the noncommercial replication and distribution of the article with the strict proviso that no changes or edits are made and the original work is properly cited (including links to both the formal publication through the relevant DOI and the license). See: https://creativecommons.org/licenses/by-nc-nd/4.0/.

\section{References}

1. Silverberg SJ, Shane E, Jacobs TP, et al. A 10-year prospective study of primary hyperparathyroidism with or without parathyroid surgery. $\mathrm{N}$ Engl J Med 1999;341:1249-55.

2. Chapman I, Horowitz M, Need AG, et al. Primary hyperparathyroidism: pathogenesis, diagnosis, and management. Compr Ther 1988;14:65-73.

3. Krempl GA, Medina JE. Current issues in hyperparathyroidism. Otolaryngol Clin North Am 2003;36:207-15

4. Boonen S, Bouillon R, Fagard K, et al. Primary hyperparathyroidism: pathophysiology, diagnosis and indications for surgery. Acta Otorhinolaryngol Belg 2001;55:119-27.

5. Taillefer R, Boucher Y, Potvin C, et al. Detection and localization of parathyroid adenomas in patients with hyperparathyroidism using a single radionuclide imaging procedure with technetium-99m-sestamibi (double-phase study). J Nucl Med 1992;33:1801-7.

6. Carling T, Udelsman R. Focused approach to parathyroidectomy. World J Surg 2008;32:1512-7.

7. Lew JI, Solorzano CC. Surgical management of primary hyperparathyroidism: state of the art. Surg Clin North Am 2009;89:1205-25.

8. Nilubol N, Beyer T, Prinz RA, et al. Mediastinal hyperfunctioning parathyroids: incidence, evolving treatment, and outcome. Am J Surg 2007;194:53-6.

Cite this article as: Deng S, Liu C, Pu Q, Liu L. Thoracoscopic resection of an ectopic mediastinal parathyroid adenoma in an octogenarian with recurrent hyperparathyroidism. Transl Cancer Res 2018;7(4):1184-1188. doi: $10.21037 /$ tcr.2018.08.27
9. Randone B, Costi R, Scatton O, et al. Thoracoscopic removal of mediastinal parathyroid glands: a critical appraisal of an emerging technique. Ann Surg 2010;251:717-21.

10. Chae AW, Perricone A, Brumund KT, et al. Outpatient video-assisted thoracoscopic surgery (VATS) for ectopic mediastinal parathyroid adenoma: a case report and review of the literature. J Laparoendosc Adv Surg Tech A 2008;18:383-90.

11. Alesina PF, Moka D, Mahlstedt J, et al. Thoracoscopic removal of mediastinal hyperfunctioning parathyroid glands: personal experience and review of the literature. World J Surg 2008;32:224-31.

12. Kuo LE, Wachtel H, Fraker D, et al. Reoperative parathyroidectomy: who is at risk and what is the risk? J Surg Res 2014;191:256-61.

13. Sukumar MS, Komanapalli CB, Cohen JI. Minimally invasive management of the mediastinal parathyroid adenoma. Laryngoscope 2006;116:482-7.

14. Prinz RA, Lonchyna V, Carnaille B, et al. Thoracoscopic excision of enlarged mediastinal parathyroid glands. Surgery 1994;116:999-1004; discussion 1004-5.

15. Cakal E, Cakir E, Dilli A, et al. Parathyroid adenoma screening efficacies of different imaging tools and factors affecting the success rates. Clin Imaging 2012;36:688-94.

16. Lumachi F, Tregnaghi A, Zucchetta P, et al. Technetium99m sestamibi scintigraphy and helical CT together in patients with primary hyperparathyroidism: a prospective clinical study. Br J Radiol 2004;77:100-3.

17. Sagan D, Gozdziuk K. Surgical treatment of mediastinal parathyroid adenoma: rationale for intraoperative parathyroid hormone monitoring. Ann Thorac Surg 2010;89:1750-5.

18. Inabnet WB. Intraoperative parathyroid hormone monitoring. World J Surg 2004;28:1212-5.

19. Patel KN, Caso R. Intraoperative Parathyroid Hormone Monitoring: Optimal Utilization. Surg Oncol Clin N Am 2016;25:91-101. 